\title{
Enhancement Technique of Infrared Images
}

Neven Sadic

\author{
Dept. of Electronics and electrical \\ communication, Faculty of electronic \\ Engineering, Menoufia University, Egypt.
}

\section{Sami El-dolil}

Dept. of Electronics and electrical
communication, Faculty of electronic
ngineering, Menoufia University, Egypt.
Emad Hassan

Dept. of Electronics and electrical

communication, Faculty of electronic

Engineering, Menoufia University, Egypt.

Moawad I. Dessoky

Dept. of Electronics and electrical

communication, Faculty of electronic

Engineering, Menoufia University, Egypt.
S. El-Rabaie

Dept. of Electronics and electrical communication, Faculty of electronic Engineering, Menoufia University, Egypt.

\section{FathiAbd El-samie}

\author{
Dept. of Electronics and electrical \\ communication, Faculty of electronic \\ Engineering, Menoufia University, Egypt.
}

\begin{abstract}
This paper presents an efficient technique for enhancement of IR images, its basic idea is to use nonlinearities applied on the main trend in IR images extracted through a LPF and the details extracted through a HPF. Magnification of the details is applied, while damping of the main trend or local mean is also accomplished to attenuate the darkness effect in IRimages. Anon-linear model is applied anther trend and gain factor is applied to details .this gain is also derived from the local mean of the image the proposed approach succeeded in quality enhancement of IR images
\end{abstract}

Keywords: Deep learning, Convolutional neural network (CNN), Normal and abnormal corneal images and model accuracy.

\section{INTRODUTION}

Infrared (IR) light is electromagnetic radiation with a wavelength longer than that of visible light, measured from the nominal edge of visible red light at 0.74 micrometers, and extending conventionally to 300micromeres. These wavelengths correspond to a frequency range of approximately 1 to $400 \mathrm{THz}$, IR images are thermal images acquired based on the heat distribution of objects this radiation can be acquired with certain thermal sensors to interpret the heat distribution as an image .unfortunately, this image is of low contrast . to acquire useful information from these images ,they need some sort of enhancement the objective of the enhancement process is to magnify the effect of object in images and eliminate the severness of the back ground traditional image enhancement teqniques such as noise reduction ,high pass filtering and median filtering are not feasible with the nature of IR images.

Different approaches have been presented for IR image enhancement Ashiba et al. presented an approach for IRimage enhancement based an hommorphic transform the idea of this approach is to decompose the image into illumination and reflectance components the dark illumination component is attenuated and the reflectance component is magnified [1] .

Ashiba et al. also suggested the utilization of the AWT for the decomposion of the IR images into an approximation and details components the detail components are enhanced with homomorphic transform and merged again with approximation component[2].

Moreover ,Ashiba et al. developed another IR enhancement technique that depends on histogram equalization and histogram matching . Donia et al.developed another method for IR image enhancement using histogram matching also. The basic idea of histogram matching is to modify the mean and variance of the poor quality image based on the mean and variance ofa good quality image this method enhances the visual quality but the enhancement may appear as being not natural .

So, there is bad need to develop more sophisticated enhancement methods that are self-confidence and that depend on the nature of the image itself not an another reference image that may not be related to the original scene of interest .This paper presents a decomposition and nonlinearity model to enhance IR images.

Infrared imaging can be used to capture images for the temperature distribution of an object. In medical applications, it is also applied in the diagnosis and prediction of several disease [3- 5], in Military applications includes target acquisition, surveillance, night-vision, homing, and tracking. Non-military uses include thermal efficiency analysis, remote temperature sensing, short-range wireless communications, spectroscopy, and weather forecasting $[6,7,8]$. IR images are created from IR radiation of objects and their backgrounds. The small temperature difference between target objects and their backgrounds results in the low contrast of these images. IR radiation is a type of electromagnetic radiation, which is not seen, but it can be felt as a heat $[9,10]$.

This paper is organized as follows: - section II, covers the problem under consideration. Section III, presents the method 
of solution followed by the image quality evaluation metrics in section IV. The results and conclusions are included in sections $\mathrm{V}$ and VI respectively.

\section{Homomorphic IR ImageEnhancement}

Images normally consist of light reflected from objects. The basic nature of the image $F\left(n_{1}, n_{2}\right)$ may be characterized by two components; the first part is the amount of source light incident on the scene being viewed, and the second part is the amount of light reflected by the objects in the scene. These portions of light are called the illumination and reflectance components, and are denoted $\mathrm{I}\left(\mathrm{n}_{1}, \mathrm{n}_{2}\right)$ and $\mathrm{R}\left(\mathrm{n}_{1}, \mathrm{n}_{2}\right)$, respectively. An image can be represented as a product of the light illumination incident on the object to be imaged and the reflectance of that object as in the following equation [9-12].

$$
F\left(n_{1}, n_{2}\right)=I\left(n_{1}, n_{2}\right) R\left(n_{1}, n_{2}\right)
$$

where $\mathrm{F}\left(\mathrm{n}_{1}, \mathrm{n}_{2}\right)$ is the obtained image pixel value, $\mathrm{I}\left(\mathrm{n}_{1}, \mathrm{n}_{2}\right)$ is the light illumination incident on the object to be imaged and $\mathrm{R}\left(\mathrm{n}_{1}, \mathrm{n}_{2}\right)$ is the reflectance of that object, where 0 $<\mathrm{I}\left(\mathrm{n}_{1}, \mathrm{n}_{2}\right)<1$ and $0<\mathrm{R}\left(\mathrm{n}_{1}, \mathrm{n}_{2}\right)<1$.

It is known that the illumination is approximately constant since the light falling on all objects is approximately the same. The only change between object images is in the reflectance component. We can think in IR images in a similar manner. For thermal IR imaging for example, an isotropic radiator for heat emits equally in all directions. So, the intensities of the image obtained for that isotropic radiator will be approximately constant, which models the illumination in visual-light imaging. If the heat distribution of the radiator is not uniform, we can model that by the multiplication of the IR radiation of an isotropic source with a detail function similar to the reflectance component in the visual-light imaging.

If we apply a logarithmic process on Eq. (1), we can change the multiplication process into an addition process as follows:

$$
\begin{aligned}
& \log \left(F\left(n_{1}, n_{2}\right)\right)= \\
& \log \left(I\left(\left(n_{1}, n_{2}\right)\right)+\log \left(R\left(n_{1}, n_{2}\right)\right)\right.
\end{aligned}
$$

The first term in the above equation has small variations, but the second term has large variations as it corresponds to the reflectivity of the object to be imaged. By attenuating the first term and reinforcing the second term of Eq. (2), we can reinforce the image details, as shown in Fig (1).

To invert the logarithmic process, we apply an exponential process. So, the Homomorphic enhanced image will be given by:

$$
g\left(n_{1}, n_{2}\right)=i^{\prime}\left(n_{1}, n_{2}\right) r^{\prime}\left(n_{1}, n_{2}\right)
$$

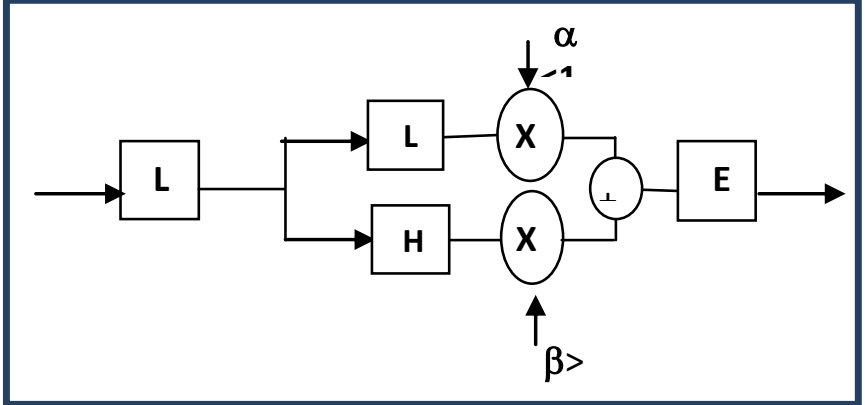

Fig.1 Homomorphic processing of an IR image.

\section{PROBLEM FORMULATION}

IR images have low contrast between background and targets and small Signal-to-Noise Ratios (SNRs). These characteristics reduce the detect ability of targets from IR images. In order to recognize targets correctly from these images good enhancement technique must be applied. The aim of image enhancement is improving the visibility of lowcontrast images and decreasing noise to obtain an image with as much details as possible [6-8]. In a variety of practical problems, it is desirable to modify the local contrast and local luminance mean. For example, when an image with a large dynamic range is recorded on a medium with smaller dynamic range, the details of the image in the very high and/or low luminance regions cannot be well represented, One approach to such a problem is a simultaneous contrast enhancement and dynamic range reduction, which can be accomplished by modification of the local contrast and the local luminance mean, One system used to modify the local contrast and the local luminance mean is illustrated in Fig.2 [13]. In our work we present an efficient technique for enhancement of IR images using this system but with a new nonlinearity then compare with the other enhancement techniques.

\section{METHOD OF SOLUTION}

In this system $\mathrm{F}(\mathrm{n} 1, \mathrm{n} 2)$ denotes the unprocessed image

$$
\mathrm{F}(\mathrm{n} 1, \mathrm{n} 2)=\mathrm{F}^{\prime}(\mathrm{n} 1, \mathrm{n} 2)+\mathrm{FH}(\mathrm{n} 1, \mathrm{n} 2)
$$

The sequence $\mathrm{FL}(\mathrm{n} 1, \mathrm{n} 2)$ which denotes the local luminance mean of $\mathrm{F}(\mathrm{n} 1, \mathrm{n} 2)$ is obtained by low pass filtering $\mathrm{F}(\mathrm{n} 1, \mathrm{n} 2)$. The local contrastFH(n1,n2) is obtained by subtracting $F L(n 1, n 2)$ from $F(n 1, n 2)$. The local contrast is modified by multiplying $\mathrm{FH}(\mathrm{n} 1, \mathrm{n} 2)$ with $\mathrm{k}$ ( $\mathrm{fL})$, which is a scalar that is a function of $\mathrm{FL}(\mathrm{n} 1, \mathrm{n} 2)$. The modified contrast is denoted by $F_{H}^{\prime}(n 1, n 2)$. If $\mathrm{k}(\mathrm{fL})$ is greater than one, the local contrast is increased, while $\mathrm{k}$ (fL) less than one represent local contrast decrease. The local luminance mean is modified by subjected to nonlinearity, and the modified local luminance mean is denoted byF'L(n1,n2). The modified local contrast and local luminance mean are then combined to obtain the processed image $\mathrm{P}(\mathrm{n} 1, \mathrm{n} 2)$.

$$
\mathrm{P}(\mathrm{n} 1, \mathrm{n} 2)=\mathrm{F}^{\prime} \mathrm{L}(\mathrm{n} 1, \mathrm{n} 2)+\mathrm{F}^{\prime} \mathrm{H}(\mathrm{n} 1, \mathrm{n} 2)
$$




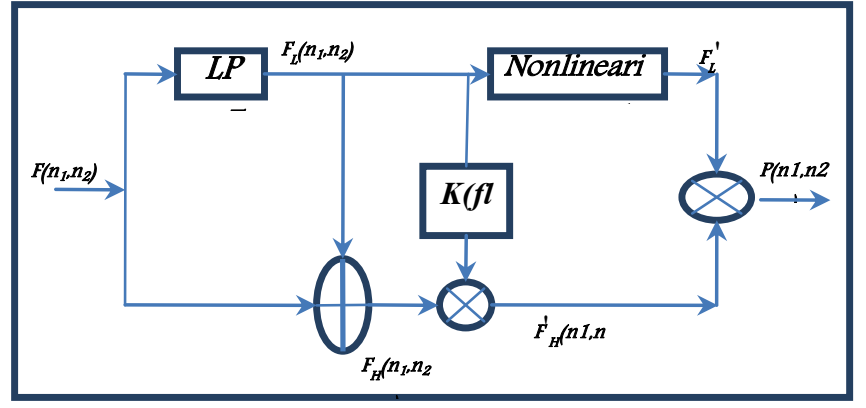

Fig. 2 System used for IR image enhancement using nonlinearity.

The system in Fig. 1 can be viewed as a special case of a two-channel process. In the two-channel process, the image to be processed is divided into two components, the local luminance means and the local contrast. The two components are modified separately and then the results are combined. In the system in Fig.1, the local luminance mean is modified by a non-linearity, and the local contrast is modified by the multiplication factor $\mathrm{k}$ ( $\mathrm{fL}$ ) function of local luminance mean [2].

The low pass filtering operation used is a simple local averaging given by [13]:-

$$
F_{L}\left(n_{1}, n_{2}\right)=\frac{1}{\left(2 N_{1}+1\right)\left(2 N_{2}+1\right)} \cdot \underset{\sum=-N_{1}+n_{1} 1=-N_{2}+n_{2}}{+N_{1}+n 1}+\sum_{2}+n_{2}{ }_{K}(k, 1)
$$

With $\mathrm{N} 1=\mathrm{N} 2=8$.

To increase the local contrast and decrease the local luminance mean when the local luminance mean is high, we choose a larger $\mathrm{k}$ (fL) for a larger fL, as shown in Fig.2

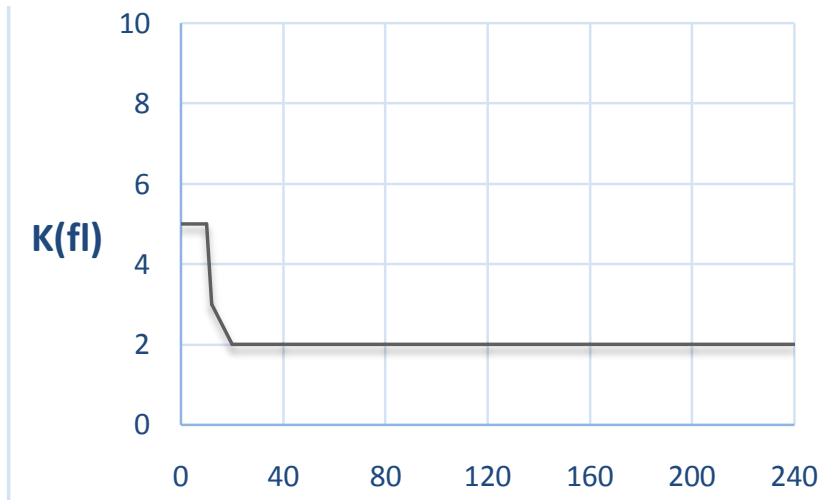

Fig. 2 Function k (fL) used in the processing [13].

Fig. 2 can be represented mathematically by this equation:

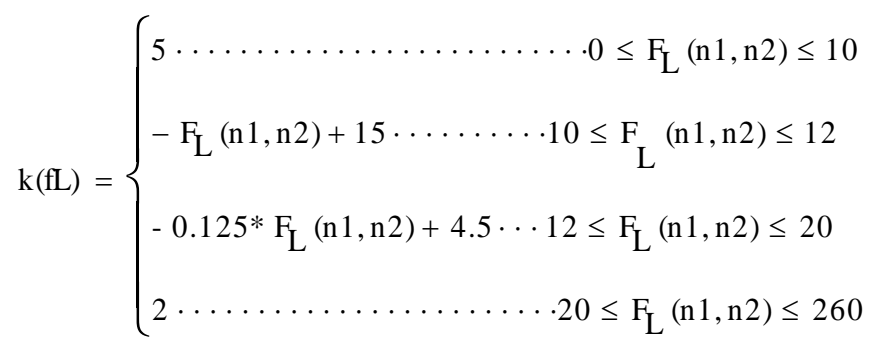

The second step in IR image enhancement system is to choose the appropriate nonlinearity, taking into account the local luminance mean change and the contrast increase as shown in Fig.3.

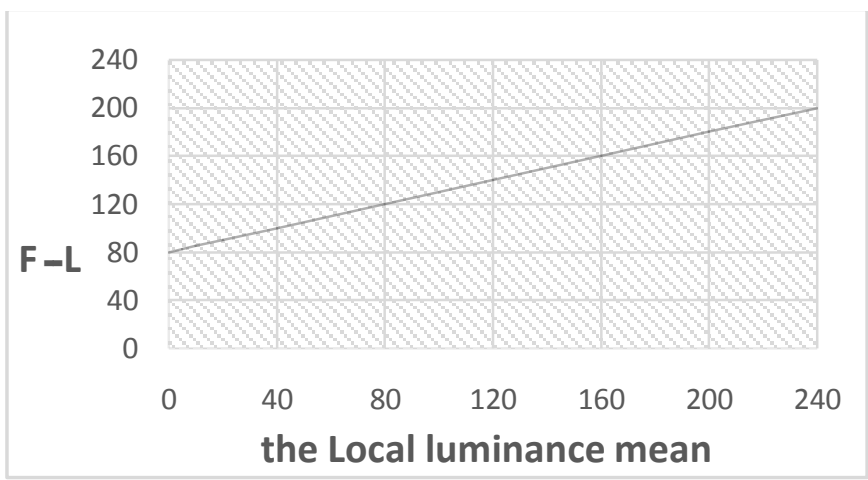

Fig.3 Nonlinearity used in the processing.

the equation that represents Fig. 3 can be expressed as :-

$F_{L}^{\prime}(n 1, n 2)=0.5 * F_{L}(n 1, n 2)+80 \quad 0<F_{L}(n 1, n 2)<240$

\section{Image Quality Evaluation Metrics}

Image quality evaluation is often used in the industry to assess the performance of imaging peripherals such as digital cameras, scanners, printers and displays. It is also used extensively by software engineers who need to investigate the performance of digital image algorithms. In our experiments, five metrics are used to evaluate the fusion results in addition to the visual quality. These metrics include entropy, average gradient, standard deviation, local contrast, and edge intensity [14].These quality metrics are discussed as follows [15].

\section{a. $\operatorname{Entropy}(E)$ :}

The entropy of the image is an important index to measure the amount of image information. It is defined as:

$$
E=-\sum_{i=O}^{N} p\left(x_{i}\right) \log p\left(x_{i}\right)
$$

where $x_{i}$ is the grayness value of the $\mathrm{i}^{\text {th }}$ point, and $p$ is the corresponding probability. Entropy reflects the average of information carried by images. The larger the entropy, the more information the image carries.

\section{b. Average Gradient ( $g$ ):}

It is the index that reflects the detail contrast and texture variation. It is calculated as: 


$$
g=\frac{1}{(M-1)(N-1)} \sum_{i=1}^{(M-1)(N-1)} \sqrt{\frac{\left(\frac{\partial f}{\partial x}\right)^{2}+\left(\frac{\partial f}{\partial y}\right)^{2}}{2}}
$$

where $M$ and $N$ are the dimensions of the image. Generally, the larger the value of $g$, the more definite the fused image.

\section{c. $\quad$ Standard Deviation (STD):}

Standard deviation is the statistical measurement that is used to determine how much variation is from the average or mean. The variance of image $P$ reflects the dispersion degree between the gray values and the gray mean value. The standard deviation is the square root of the variance. The higher the standard deviation, the clearer the fused image. It is defined as:

$$
S T D=\sqrt{\frac{\sum_{i=1}^{M} \sum_{j=1}^{N}|P(i, j)-\mu|^{2}}{M N}}
$$

where $P(i, j)$ is the image, $M$ and $N$ are the dimensions of the image and $\mu$ is the mean value.

\section{d. Local Contrast $\left(C_{\text {local }}\right)$ :}

It is very important for target detection, recognition, and tracking tasks, and it is defined as:

$$
C_{\text {Local }}=\frac{\left|\mu_{\text {targ et }}-\mu_{\text {background }}\right|}{\mu_{\text {targ et }}+\mu_{\text {background }}}
$$

where, $\mu_{\text {targ et }}$ and $\mu_{\text {background }}$ are the means of the target and the background in a local region. It is known that, as the image local contrast increases, the quality of the image increases and a better view of the image is achieved.

\section{e. $\quad$ Edge Intensity (S):}

One of the simplest and most widely used assistant tools for image evaluation is the edge detection. The Sobel operator can be used for edge detection, and it is characterized by its simplicity and good performance.

In the Sobel edge detection, the input image $P$ is convolved with two filters; $h_{x}$ and $h_{y}$.

$$
h_{x}=\left(\begin{array}{rrr}
1 & 0 & 1 \\
-2 & 0 & 2 \\
-1 & 0 & 1
\end{array}\right) h_{y}=\left(\begin{array}{rrr}
-1 & -2 & -1 \\
0 & 0 & 0 \\
1 & 2 & 1
\end{array}\right)
$$

where the filter $h_{x}$ corresponds to horizontal differentiation with respect to vertical edges, while the filter $h_{y}$ corresponds to vertical differentiation with respect to horizontal edges.
The results of the two convolutions are combined in the following way to obtain the Sobel edge intensity $(S)$. It is known that the higher $\mathrm{S}$ the more image details are.

$$
\begin{aligned}
& S_{x}=P * h_{x} \quad S y=P * h_{y} \\
& S=\sqrt{\left(S_{x}^{2}+S_{y}^{2}\right)}
\end{aligned}
$$

\section{Results}

The following figures (3-7) and tables (1-4) show the results of applying the system in Fig. 1 to enhance different IR images. The original image of $(256 \times 256)$ pixels. We have applied the system with four different IR images and evaluate the five metric (entropy, Average Gradient, Standard Deviation, Local Contrast, Edge Intensity) for each in state of the proposed work, adaptive wavelet and hommomorphic enhancement techniques. From the Figures and the tables above, it is clear that the Proposed technique is the most appropriate approach for enhancement of IR images compared with the other approaches

\section{Conclusion}

In this paper, we have implemented different IR image enhancement methods. The main target is to obtain IR images with more details and improving visibility. Simulation results have shown that the proposed technique gives more details and good enhancement compared with the other techniques 


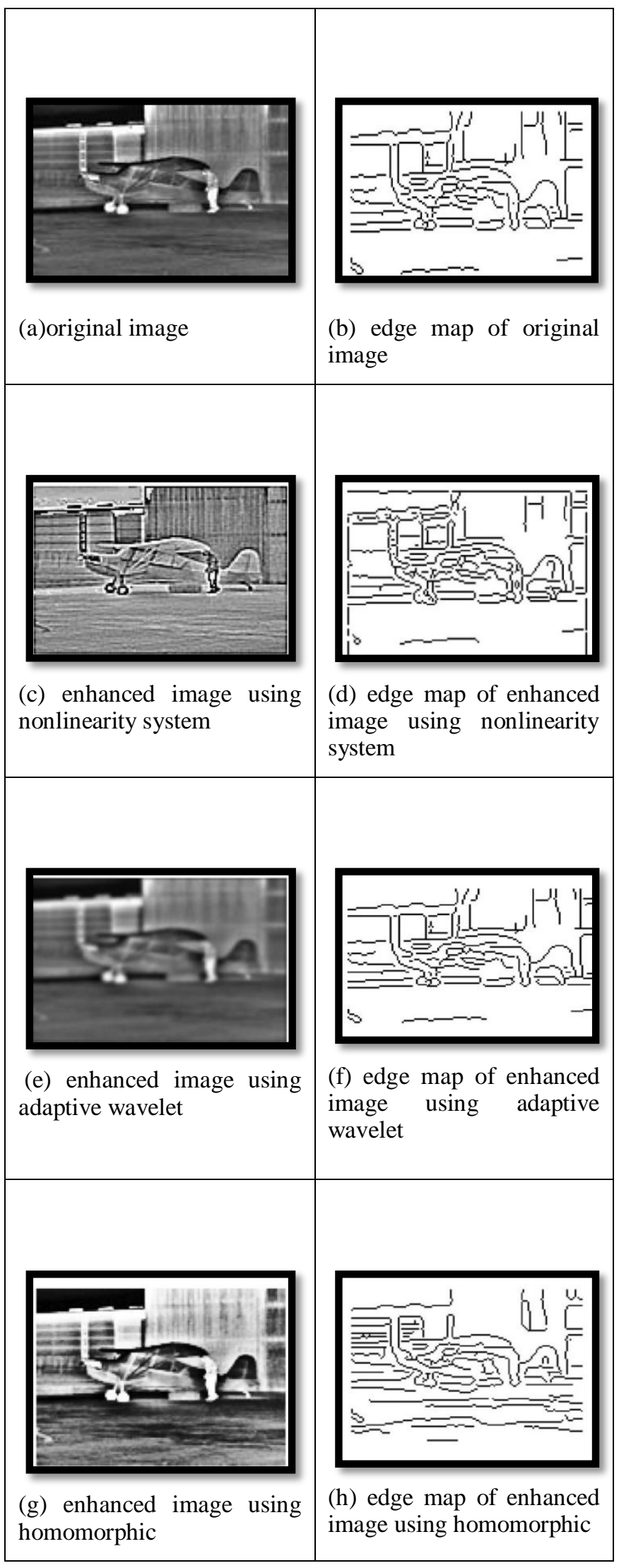

Fig. 4 Plane image with the 3 different methods for enhancement and its maps.

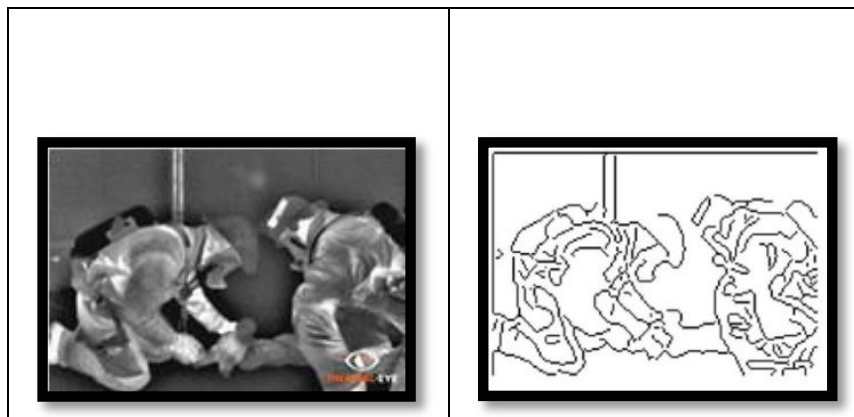

(a) original image

(b) edge map of original image

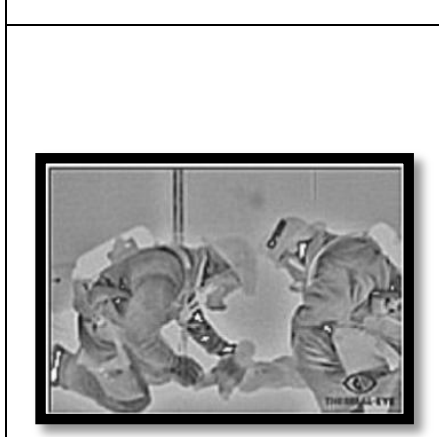

(c)enhanced image using nonlinearity system

(d) edge map of enhanced image using nonlinearity

system
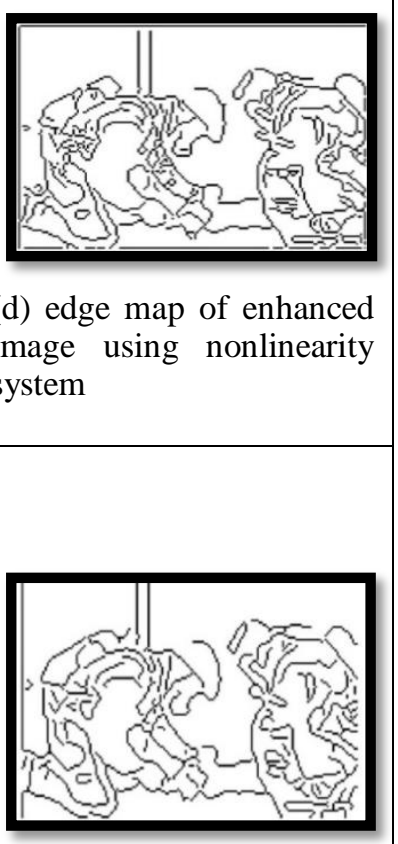

(e) enhanced image using adaptive wavelet

(f) edge map of enhanced image using adaptive wavelet

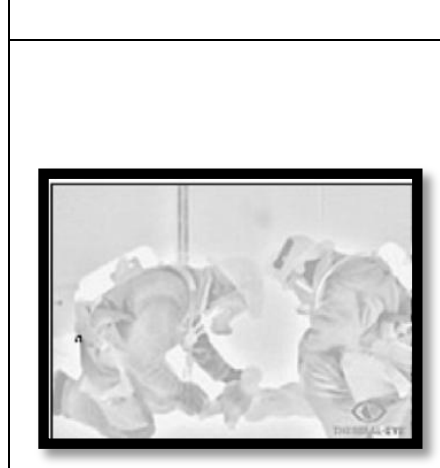

(g) enhanced image using homomorphic

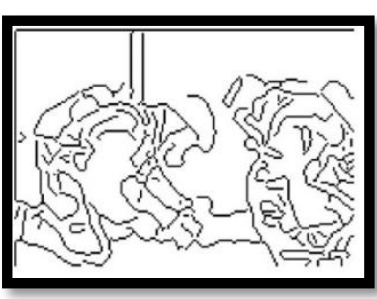

(h) edge map of enhanced image using homomorphic

Fig.5 Fire man image with 3 different methods for enhancement and its maps. 


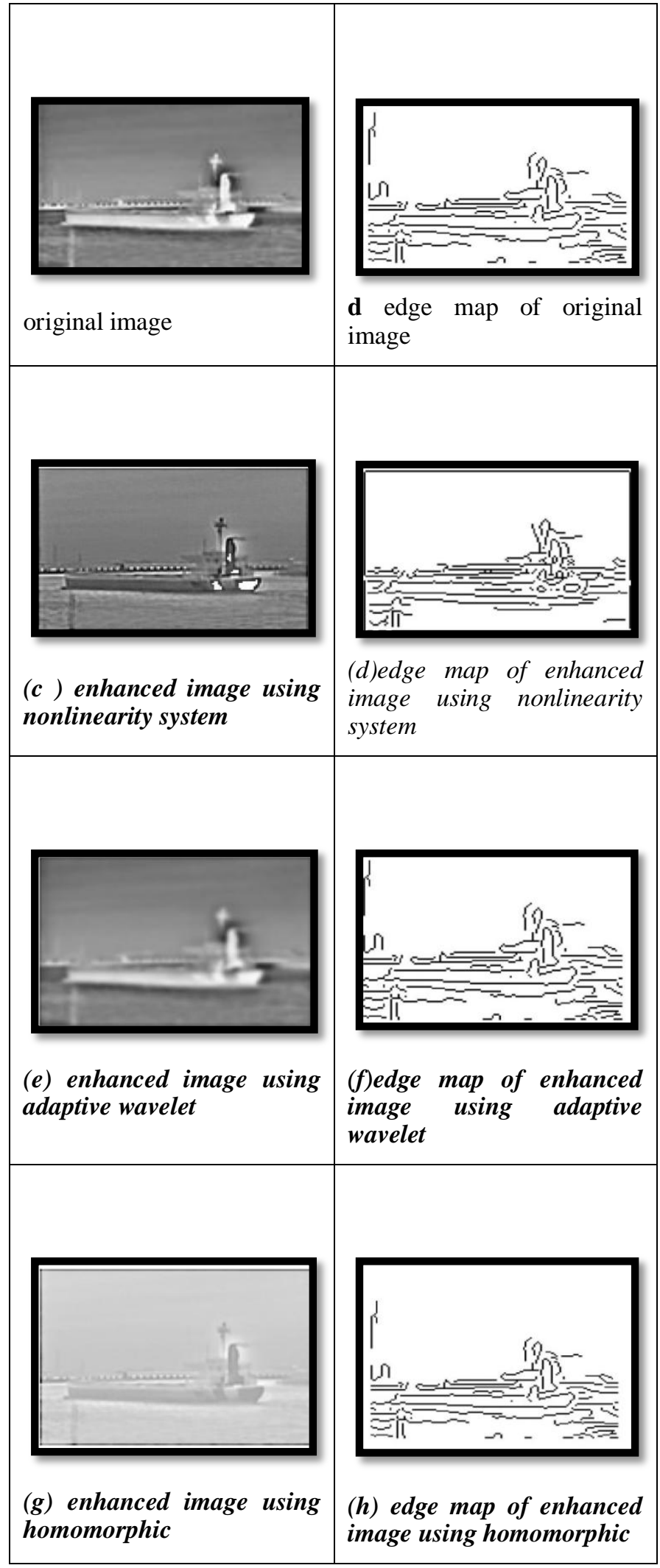

Fig. 6 Boat image with 3 different methods for enhancement and its maps.

\section{Numerical Results}

Table 1: Evaluation metrics results for plane image.

\begin{tabular}{|l|c|c|c|c|c|}
\hline $\begin{array}{l}\text { Metric } \\
\text { Model used }\end{array}$ & $\mathrm{E}$ & $\mathrm{g}$ & $\mathrm{STD}$ & $\left(\mathrm{C}_{\text {local }}\right)$ & $\mathrm{S}$ \\
\hline $\begin{array}{l}\text { Additive } \\
\text { wavelet }\end{array}$ & 0 & 0.7107 & 0.2213 & 0.0302 & 7.9201 \\
\hline Homomorphic & 0.020 & 1.5660 & 0.2083 & 0.0552 & 17.1138 \\
\hline $\begin{array}{l}\text { Proposed } \\
\text { technique }\end{array}$ & 0.0801 & 2.4039 & 0.1777 & 0.0656 & 26.1760 \\
\hline
\end{tabular}

Table 2 : Evaluation metrics results for Fire man image.

\begin{tabular}{|l|c|c|c|c|c|}
\hline $\begin{array}{l}\text { Metric } \\
\text { Model used }\end{array}$ & $\mathrm{E}$ & $\mathrm{g}$ & $\mathrm{STD}$ & $\mathrm{C}_{\text {local }}$ & $\mathrm{S}$ \\
\hline $\begin{array}{l}\text { Additive } \\
\text { wavelet }\end{array}$ & 0 & 0.8804 & 0.2379 & 0.0391 & 9.7692 \\
\hline Homomorphic & 0.0230 & $\mathbf{0 . 9 0 5 4}$ & $\mathbf{0 . 0 8 0 7}$ & $\mathbf{0 . 0 2 5 8}$ & 9.9243 \\
\hline $\begin{array}{l}\text { Proposed } \\
\text { technique }\end{array}$ & 0.0296 & 1.9314 & $\mathbf{0 . 1 9 9 4}$ & $\mathbf{0 . 0 5 6 8}$ & 20.7590 \\
\hline
\end{tabular}

Table 3 : Evaluation metrics results for Boat image.

\begin{tabular}{|l|l|l|l|l|l|}
\hline \multicolumn{1}{|c|}{ Metric } & $\mathrm{E}$ & $\mathrm{g}$ & $\mathrm{STD}$ & $\mathrm{C}_{\text {local }}$ & $\mathrm{S}$ \\
\hline Additive wavelet & 0 & 0.5053 & 0.1724 & 0.0202 & 5.6478 \\
\hline Homomorphic & $\begin{array}{l}4.3239 \mathrm{e} \\
-004\end{array}$ & 0.4987 & 0.0801 & 0.0159 & 5.5406 \\
\hline $\begin{array}{l}\text { Proposed } \\
\text { technique }\end{array}$ & 0.0233 & 0.8766 & 0.1865 & 0.0301 & 9.6415 \\
\hline
\end{tabular}

*** (E) - Entropy

* (STD)-Standard Deviation

***** (S) - Edge Intensity

\section{References}

[1]A. Cohen and J. Kovacevec, "Wavelets: The Mathematical Background," Proceedings of the IEEE, vol. 84, no. 4, pp. 514- 522, April 1996

[2] J. S. Lim, Two-Dimensional Signal and Image Processing. Prentice Hall Inc., 1990.

[3] Z. H. Long, " Image Fusion Using Wavelet Transform," in Proc. The Symposium on Geospatial Theory, Processing and Applications, 2002.

[4] V. S. Petrovic and C. S. Xydeas, " Gradient-Based Multiresolution Image Fusion," IEEE Trans. Image Processing, vol. 13, no. 2, pp. 228-237, February 2004.

[5] I. Daubechies, "Where Do Wavelets Come From?-A Personal Point of View," Proceedings of the IEEE, vol. 84, no. 4, pp. 510- 513, April 1996.

[6] B. Zitova and J. Flusser, "Image Registration Methods: A Survey," Image and Vision Computing, vol. 21, pp. 977-1000, 2003.

[7] M. Xia, and B. Liu, "Image Registration by Super Curves," IEEE Trans. Image Processing, vol. 13, no. 5, pp.720-732, May 2004.

[8] D. Robinson and P. Milanfar, "Fundamental Performance Limits in Image Registration,” vol. 13, no.9, pp. 1185-1199, September 2004.

[9] W. K. Pratt, Digital Image Processing. John Wiley \& Sons Inc., 1991.

[10] H. Li, B. S. Manjunath and S. K. Mitra, " Multi-Sensor Image Fusion Using The Wavelet Transform,” in Proc. ICIP, pp. 51-55, 1994. 
[11] G. Piella and H. Heijmans, “ Multiresolution Image Fusion Guided By A Multimodal Segmentation," in Proc. ACIVS, pp. 1- 8, 2002.

[12] J. H. Shin, J. H. Jung, J.K. Paik and M. A. Abidi, “ Data Fusion-Based Spatio-Temporal Adaptive Interpolation For Low-Resolution Video," in Proc. ICIP , 2001.

[13] Tamer Prli and Jae S. Lim, "Adaptive Filtering for Image Enhancement", 1981 IEEE, pp. 1117-1120. .

[14] T. T. Neo, "Fusion of Night Vision and Thermal Images', M.Sc. Thesis, Naval Postgraduate School, University of New South Wales, Australia, 2006.

[15] S. Yin, L. Cao, Q. Tan, and G. Jin, "Infrared and Visible Image Fusion based on NSCT and Fuzzy Logic ", in Proc. of the IEEE Int. Conf. on Mechatronics and Automation, pp. 671-675, August 4-7, 2010. 